International Journal of Pure and Applied Mathematics

Volume 103 No. 3 2015, 395-401

ISSN: 1311-8080 (printed version); ISSN: 1314-3395 (on-line version)

url: http://www.ijpam.eu

doi: http://dx.doi.org/10.12732/ijpam.v103i3.2

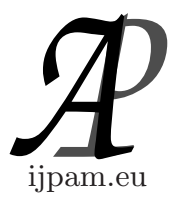

\title{
SOME PROPERTIES ON FLM ALGEBRAS
}

\author{
A. Naziri-Kordkandi ${ }^{1 \S}$, A. Zohri ${ }^{2}$, F. Ershad ${ }^{3}$, B. Yousefi ${ }^{4}$ \\ 1,2,3,4 Department of Mathematics \\ Payame Noor University \\ P.O. Box 19395-3697, Tehran, IRAN
}

\begin{abstract}
In this paper, we extend some results of Banach algebras concerning the radical and spectral properties to the fundamental locally multiplicative topological algebras (FLM algebras).
\end{abstract}

AMS Subject Classification: $46 \mathrm{H} 05$

Key Words: fundamental topological algebra, FLM algebra, spectrum, spectral radius, radical

\section{Introduction}

The fundamental topological spaces (also algebras) has been introduced in [1] in 1990 extending the meaning of locally bounded and locally convex algebras. Some basic theorems are proved on fundamental topological vector spaces and fundamental topological algebras in [1],[2],[3],[4].

The fundamental locally multiplicative topological algebras (abbreviated by FLM) with a property very similar to the normed algebras, is also introduced in [3], and some of famous theorems of Banach algebras are extended to complete metrizable FLM algebras. In this note, we generalize some properties of Banach algebras concerning the spectral properties and radical to the FLM algebras.

Received: February 20, 2015

(C) 2015 Academic Publications, Ltd. url: www.acadpubl.eu

$\S_{\text {Correspondence author }}$ 


\section{Difinitions}

In this section, we state a number of definitions of terms which we shall use throughout this paper.

Definition 1. Let $A$ be a topological linear space.

$A$ is said to be fundamental one if there exists $b>1$ such that for every sequence $\left\{x_{n}\right\}$ of $A$, the convergence of $b^{n}\left(x_{n}-x_{n-1}\right)$ to zero in $A$ implies that $\left\{x_{n}\right\}$ is Cauchy.

A fundamental topological algebra is an algebra whose underlying topological linear space is fundamental.

Definition 2. A fundamental topological algebra is called to be locally multiplicative, if there exists a neighbourhood $U_{0}$ of zero such that for every neighbourhood $V$ of zero, the sufficiently large powers of $U_{0}$ lie in $V$. We call such an algebra, an FLM algebra.

Definition 3. Let $\left(A, d_{A}\right)$ be a metrizable topological algebra. we say that $A$ is a submultiplicative metrizable topological algebra if

$$
d_{A}(0, x y) \leq d_{A}(0, x) d_{A}(0, y), \quad \forall x, y \in A
$$

Definition 4. Let $A$ be a complete metrizable FLM algebra. An element $x \in A$ is called quasinilpotent if $x$ satisfies $r(x)=0$ (and hence $s p(x)=\{0\}$ ). Note that $r(x)$ is the spectral radius of $x$ and defined by

$$
r(x)=\sup \{|\lambda|: \lambda \in \operatorname{sp}(x)\}
$$

We need the following known result in the next section. See, for example [7].

Corollary 5. If $A$ is a unital algebra, then

$$
\operatorname{Rad}(A)=\{x \in A: r(a x)=0, \quad \forall a \in A\},
$$

where $\operatorname{Rad}(A)$ is the radical of $A$.

Definition 6. Let $A$ be a complete metrizable FLM algebra and let $\pi$ : $A \rightarrow A / \operatorname{Rad}(A)$ be the quotient map. The centre modulo the radical of $A$ is defined by

$$
S(A)=\pi^{-1}[Z(A) / \operatorname{Rad}(A)]=\{x \in A: x a-a x \in \operatorname{Rad}(A), \forall a \in A\}
$$

where $Z(A)$ is the centre of $A$. 
Definition 7. A unital linear map $\varphi$ from a unital complete metrizable FLM algebra $A$ to a unital complete metrizable FLM algebra $B$ is said to be preserve invertibility if $\varphi(x) \in \operatorname{Inv}(B)$ whenever $x \in \operatorname{Inv}(A)$, the set of invertible elements of $A$.

Definition 8. Let $A$ and $B$ be complete metrizable FLM algebras. A linear map $\varphi: A \rightarrow B$ is said to be:

i) spectrum compressing if for all $x \in A, s p(\varphi(x)) \subseteq s p(x)$.

ii) spectrum preserving if for all $x \in A, s p(\varphi(x))=s p(x)$.

iii) spectral isometry if for all $x \in A, \quad r(\varphi(x))=r(x)$

\section{New Results on FLM Algebras}

On FLM algebras with a property similar to the Banach algebras, some theorems and results have already been obtained. In this section, we continue this process and get some new results concerning the spectral properties and radical on FLM algebras.

Theorem 9. Let $A$ and $B$ be complete metrizable FLM algebras and let $\varphi: A \rightarrow B$ be a linear map. Then the followings hold:

i) If $\varphi$ is spectrum preserving, then $\varphi$ is spectral isometry.

ii) If $\varphi$ is spectrum compressing, then $\varphi$ preserves invertibility.

iii) If $\varphi$ is surjective and spectrum preserving, then $\varphi(\operatorname{Inv}(A))=\operatorname{Inv}(B)$.

Proof. (i) We deduce from the definition that a spectrum preserving linear map is a spectral isometry.

(ii) Let $a \in \operatorname{Inv}(A)$, then $0 \in C \backslash s p(a) \subseteq C \backslash s p(\varphi(a))$. Hence $\varphi(a) \in$ $\operatorname{Inv}(B)$.

(iii) Let $b \in \operatorname{Inv}(B)$, then $b=\varphi(a)$ for some $a$ in $A$. Since $0 \in C \backslash \operatorname{sp}(b)=$ $C \backslash s p(\varphi(a))=C \backslash s p(a)$, we conclude that $a \in \varphi(\operatorname{Inv}(A))$ and so

$$
b \in \varphi(\operatorname{Inv}(A)) .
$$

Theorem 10. Let $A$ and $B$ be commutative complete metrizable FLM algebras with unit elements $e_{A}, e_{B}$ respectively, and let $B$ be semisimple.If $\varphi: A \rightarrow B$ is a unital invertibility preserving map, then $\varphi$ is spectrum compressing.Moreover if $\varphi$ is bijective with $\varphi(\operatorname{Inv}(A))=\operatorname{Inv}(B)$, then $\varphi$ is spectrum preserving. 
Proof. Since $\varphi: A \rightarrow B$ is invertibility preserving map, then we have $\varphi(a) \in$ $\operatorname{Inv}(B)$ whenever $a \in \operatorname{Inv}(A)$. Let $\theta \in \phi_{B}$ (maximal ideal space of $B$ ) then

$$
\theta o \varphi(a)=\theta(\varphi(a)) \neq 0 \text { and } \theta o \varphi\left(e_{A}\right)=1
$$

this implies that $\operatorname{ker} \theta o \varphi \subseteq \operatorname{sing} A$. By Gleason-Kahane-Zelazko theorem in FLM algebra [4,5.5], we conclude that $\theta \circ \varphi$ is multiplicative. Hence for $x, y \in A$, we obtain

$$
\theta[\varphi(x y)-\varphi(x) \varphi(y)]=\theta o \varphi(x y)-\theta o \varphi(x) \theta o \varphi(y)=0
$$

Since $B$ is semisimple and $\theta \in \phi_{B}$ is arbitrary, it follows that

$$
\varphi(x y)=\varphi(x) \varphi(y)
$$

therefore $\varphi$ is multiplicative. By $[11,2.2], \varphi$ is spectrum compressing, i.e.

$$
s p(\varphi(x)) \subseteq s p(x) \text { for all } x \text { in } A \text {. }
$$

If $\varphi$ is bijective with $\varphi(\operatorname{Inv}(A))=\operatorname{Inv}(B)$, then $\varphi^{-1}$ is also a unital invertibility preserving map. So $s p\left(\varphi^{-1}(b)\right) \subseteq s p(b)$ for $b$ in $B$. Taking $b=\varphi(x)$ implies that $\operatorname{sp}(x) \subseteq \operatorname{sp}(\varphi(x))$.

Theorem 11. Let $A$ and $B$ be commutative complete metrizable FLM algebras with unit elements and let $B$ be semisimple.If $\varphi: A \rightarrow B$ is a unital spectrum compressing map, then the followings hold:

i) If $p$ is an idempotent, then $\varphi(p)$ is an idempotent.

ii) $\varphi$ maps the center $Z(A)$ of $A$ onto the center $Z(B)$ of $B$.

iii) $\varphi$ is continuous.

Proof. By [11,2.1], $\varphi$ is multiplicative linear map and hence (i) and (ii) follows easily.For (iii), the continuity of $\varphi$ follows from[2,4.5] and similar proof of Silove theorem in $[8,2.3 .3]$.

Here we give some useful characterization of radical (or more exactly Jacobson radical) on FLM algebras.

Theorem 12. Let $A$ be a complete metrizable FLM algebra with submultiplicative meter $d_{A}$ and unit element $e$. Then

$$
\operatorname{Rad}(Z(A))=Z(A) \cap \operatorname{Rad}(A)
$$


Proof. Let $x \in Z(A) \cap \operatorname{Rad}(A)$. Then by [5,3.1.3] , $e-a x \in \operatorname{Inv}(A)$ for all $a \in A$. Inparticular, if $a \in Z(A)$, then $e-a x \in Z(A)$ and so $(e-a x)^{-1} \in Z(A)$. By [5,3.1.3], $x \in \operatorname{Rad}(Z(A))$ and therefore $Z(A) \cap \operatorname{Rad}(A) \subseteq \operatorname{Rad}(Z(A))$.

Coversely, let $x \in \operatorname{Rad}(Z(A))$. Then $x \in Z(A)$ and $r(x)=0$ by Corollary 5.Since $x a=a x, \forall a \in A$, then $(a x)^{n}=a^{n} x^{n}$ for all $n$. By [11,3.6], we have $r(a x) \leq r(a) r(x)$. This implies that $r(a x)=0, \quad \forall a \in A$.By Corollary $5, x \in \operatorname{Rad}(A)$. But $x \in Z(A)$, thus $\operatorname{Rad}(Z(A)) \subseteq Z(A) \cap \operatorname{Rad}(A)$, which completes the proof.

Corollary 13. If $A$ is semisimple with the conditions of Theorem 12, then so is $Z(A)$.

Proof. It is clear.

Theorem 14. Let $A$ and $B$ be a complete metrizable FLM algebras and $A$ with submultiplicative meter $d_{A}$.If $\varphi: A \rightarrow B$ is a spectral isometry, then $\operatorname{ker} \varphi \subseteq \operatorname{Rad}(A)$.

Proof. Let $x \in \operatorname{ker} \varphi$. Then for each $y \in A$, we have

$$
r(y)=r(\varphi(y))=r(\varphi(x+y))=r(x+y)
$$

Inparticular, if $y \in A$ is quasinilpotent, then $0=r(y)=r(x+y)$. By $[11,3.4]$, $x \in \operatorname{Rad}(A)$ and so $\operatorname{ker} \varphi \subseteq \operatorname{Rad}(A)$.

Corollary 15. If $A$ is semisimple with the conditions of Theorem 14, then $\varphi$ must be injective.

Proof. Since $A$ is semisimple, then $\operatorname{Rad}(A)=\{0\}$ and so $\operatorname{ker} \varphi=\{0\}$. Thus $\varphi$ is injective.

Theorem 16. Suppose $A$ is a commutative complete metrizable FLM algebra with unit element e.If $\left\{x_{n}\right\}$ is any sequence of $\operatorname{Inv}(A)$ such that the set $\left\{x_{n}^{-1}: n \in N\right\}$ is bounded, then $\operatorname{Rad}(A)=\{0\}$.

Proof. At first, we show that $\operatorname{Inv}(A)$ is an open and closed subset of $A \backslash\{0\}$. Let $x \in \overline{\operatorname{Inv}(A)}$, then there is a sequence $\left\{x_{n}\right\} \subseteq \operatorname{Inv}(A)$ such that $x_{n} \rightarrow x$, by $[3,3.5], x$ is invertible and hence $\operatorname{Inv}(A)$ is closed set and By[2,4.3], it is open.Thus $\operatorname{Inv}(A)$ is an open and closed subset of $A \backslash\{0\}$. Since $A \backslash\{0\}$ is connected, therefore $\operatorname{Inv}(A)$ is either empty or $A \backslash\{0\}$ itself, but it isn't empty, since $e \in \operatorname{Inv}(A)$, Consquently $\operatorname{Inv}(A)=A \backslash\{0\}$. Hence $A$ is a division algebra and so $\operatorname{Rad}(A)=\{0\}$. 
The following theorem is a version of V.Ptak's theorem in [9] for FLM algebras.

Theorem 17. Let $A$ be a complete metrizable FLM algebra with submultiplicative meter $d_{A}$ and unit element $e$. then the following statements are equivalent:

i) $x \in S(A)$;

ii) $r(x+a) \leq r(x)+r(a)$ for all $a \in A$;

iii) $r(x a) \leq r(x) r(a)$ for all $a \in A$;

iv) $\sup \left\{r\left(x-e^{-a} x e^{a}\right): a \in A\right\}<\infty$

Proof. if necessary, we may suppose without loss of generality that $A$ is semisimple, Then (i) implies (ii) and (iii) by [11,3.6]. The similar proof of V.Ptak's theorem in [9] shows that each of (ii) and (iii) implies (iv) and also (iv) implies (i). The details can be found in [9] , [5].

Theorem 18. Let $A$ and $B$ unital complete metrizable FLM algebras with submultiplicative meters $d_{A}$ and $d_{B}$ respectively. If $\varphi: A \rightarrow B$ is a surjective spectral isometry, then

$$
\varphi[S(A)]=S(B)
$$

Proof. Let $x \in S(A)$ and $y \in B$ be arbitrary. Since $\varphi$ is surjective, then $\varphi(a)=y$ for some $a \in A$. Since $\varphi$ is spectral isometry, we have

$$
r(\varphi(x)+y)=r(\varphi(x+a))=r(x+a)
$$

By Theorem 17, we have

$$
r(\varphi(x)+y) \leq r(x)+r(a)=r(\varphi(x))+r(\varphi(a))=r(\varphi(x))+r(y)
$$

By Theorem 17, again $\varphi(x) \in S(B)$. This implies that $\varphi[S(A)] \subseteq S(B)$. For the reverse inclusion, let $y \in S(B)$. Then $y=\varphi(x)$, for some $x$ in $A$. For each $a$ in $A$, we have

$$
r(x+a)=r(\varphi(x+a))=r(y+\varphi(a))
$$

By Theorem 17, we obtain,

$$
r(x+a) \leq r(y)+r(\varphi(a))=r(x)+r(a)
$$

Hence $x \in S(A)$ and so $y \in \varphi[S(A)]$. 
Corollary 19. If $A$ and $B$ are semisimple with the conditions of Theorem 18 , then $\varphi[Z(A)]=Z(B)$.

Proof. Since $A$ and $B$ are semisimple, then $S(A)=Z(A)$ and $S(B)=$ $Z(B)$. Consequently, $\varphi[Z(A)]=Z(B)$.

\section{Acknowledgments}

The authors would like to thank the refree for helpful suggestions.

\section{References}

[1] E. Ansari-Piri, A class of factorable topological algebras, Proc. Edin. Math. Soc., 33 (1990), 53-59.

[2] E. Ansari.Piri, Topics on fundamental topological algebras, Honam math. J., 23 (2001), 59-66.

[3] E. Ansari-Piri, Topics on locally multiplicative fundamental topological algebras, Far East. J. Math. Sci. (FJMS), 8, No. 1 (2003), 77-82.

[4] E. Ansari-Piri, The linear functional on locally multiplicative topological algebras, Turk. J. Math., 34 (2010), 385-391.

[5] B. Aupetit, A Primer on Spectral Theory, Springer- Verlag, 1991.

[6] V.K. Balachandran, Topological Algebra, New York, Elsevier, 2000.

[7] F.F. Bonsal, J. Doncan, Complete Normed Algebras, Springer-Verlag, Berlin 1937.

[8] H.G. Dalse, Banach Algebras and Automatic Continuity, Clarendon Press, Oxford, 2000.

[9] V. Ptak, Derivations, commutators and the radical, Manuscripta Math., 23 (1979), 355-362.

[10] W. Rudin, Functional Analysis, McGraw- Hill, 1973.

[11] A. Zohri, A. Jabari, Generalization of some properties of Banach algebras to fundamental locally multiplicative topological algebras, Turk. J. Math., 35 (2011), 1-7. 
